\title{
Abnormal binding and disruption in large scale networks involved in human partial seizures
}

\author{
Fabrice Bartolomei ${ }^{1,2,3^{*}}$, Maxime Guye $e^{2,3,6,7}$ and Fabrice Wendling ${ }^{4,5}$
}

\footnotetext{
* Correspondence:

fabrice.bartolomei@ap-hm.fr

${ }^{1}$ INSERM, U1106, Marseille F-13000,

France

${ }^{2}$ Faculté de Médecine, Aix Marseille Université, Marseille F-13000, France Full list of author information is

available at the end of the article
}

\begin{abstract}
There is a marked increase in the amount of electrophysiological and neuroimaging works dealing with the study of large scale brain connectivity in the epileptic brain. Our view of the epileptogenic process in the brain has largely evolved over the last twenty years from the historical concept of "epileptic focus" to a more complex description of "Epileptogenic networks" involved in the genesis and "propagation" of epileptic activities. In particular, a large number of studies have been dedicated to the analysis of intracerebral EEG signals to characterize the dynamic of interactions between brain areas during temporal lobe seizures. These studies have reported that large scale functional connectivity is dramatically altered during seizures, particularly during temporal lobe seizure genesis and development. Dramatic changes in neural synchrony provoked by epileptic rhythms are also responsible for the production of ictal symptoms or changes in patient's behaviour such as automatisms, emotional changes or consciousness alteration. Beside these studies dedicated to seizures, large-scale network connectivity during the interictal state has also been investigated not only to define biomarkers of epileptogenicity but also to better understand the cognitive impairments observed between seizures.
\end{abstract}

Keywords: Synchrony, Focal epilepsies, Non linear correlation, Functional connectivity, Intracerebral EEG

\section{Review}

Approximately $30 \%$ of focal epilepsies are resistant to antiepileptic drugs. In this situation, surgical resection of the epileptogenic zone (EZ) is the only therapeutic option able to suppress seizures. The localisation and the definition of the EZ are therefore crucial issues that can be addressed through detailed analysis of anatomo-functional data acquired in epileptic patients during pre-surgical evaluation. From a theoretical viewpoint, the EZ is a highly illustrative example of complex system exhibiting nonlinear dynamics as well as ruptures (more or less abrupt) between these dynamics (typically during the transition from interictal to ictal activity) as reflected by signals directly recorded from involved brain structures.

Several reviews dealing with "neural networks" and epilepsy or with synchrony and epilepsy [1,2] are available in the literature. With regard to these reviews, our objectives were more specifically to focus on works studying functional connectivity from stereotactic EEG (SEEG) signals, to propose a general framework ("the epileptogenic

(c) 2013 Bartolomei et al.; licensee Springer on behalf of EPJ. This is an Open Access article distributed under the terms of the Creative Commons Attribution License (http://creativecommons.org/licenses/by/2.0), which permits unrestricted use, distribution, and reproduction in any medium, provided the original work is properly cited. 
network concept") and to show how this concept may give clues in the comprehension of some clinical manifestations encountered in partial epilepsies.

Therefore we concentrate on studies from our group dealing with the analysis of synchronization processes between distant structures recorded by intracerebral electrodes during seizure genesis and development. These data have been mainly obtained in the context of presurgical evaluation of temporal lobe epilepsy (TLE) and based on the analysis of intracerebral depth-EEG signals. Based on the estimation of interdependences (i.e. statistical coupling) between signals recorded from distinct sites, some reports have demonstrated that the areas involved in the generation of seizures (defining the EZ) are characterized by synchronous oscillations at seizure onset $[1,3,4]$. Other studies based on nonlinear associations in multivariate signals $[5,6]$ have also reported that large scale functional connectivity is dramatically altered during seizures, or indicated that the topology of networks changes as ictal activity develops $[7,8]$.

We will also discuss data showing that abnormal changes in neural synchrony provoked by epileptic rhythms may be responsible for (or at least be in relation with) the production of ictal symptoms or changes in patient's behaviour. Finally, recent works suggest that even at rest during the interictal period these networks are associated with changes in functional connectivity. We will illustrate these findings by discussing results we obtained using both intracerebral EEG recordings and resting-state functional MRI.

\section{The epileptogenic zone network}

The EZ may be considered as a general notion encompassing different conditions.

Regarding the organization of the EZ, two schematically-different situations may be observed when intracerebral recordings and notably when stereotactic EEG (SEEG) is used to record seizures. In some cases, the EZ corresponds to a relatively restricted area of the brain. Seizure genesis takes place in a unique "functional area", a situation corresponding to the classical notion of an epileptogenic focus, the most popular concept in epileptology (see [9]).

However, in a large number of cases (and usually in temporal lobe seizures), the seizure onset is characterized by discharges that rapidly involve several distinct brain regions [3,10]. In this situation, the model of the epileptogenic focus is too simplistic and cannot accurately describe the spatial organization of the EZ. The propagation of seizures is also a very complex process. It is indeed usual to observe that partial seizures involve several cortical and subcortical structures during their time course, sometimes with a remarkable reproducibility from one seizure to the other, in a given patient [11,12]. Figure 1 shows one example of "distributed" areas generating seizures in a case of TLE. In this example, the seizures start simultaneously from at least 3 distinct regions of the temporal lobe. Secondary delayed electrophysiological changes are observed in regions outside the EZ.

Several years ago, we have proposed that the EZ corresponds to a network of hyperexcitable structures sharing at least two main spatio-temporal characteristics $[3,10]$ :

- the ability to generate fast oscillations, in the beta or/and the gamma range, (classically called low voltage rapid discharges, LVRD). 


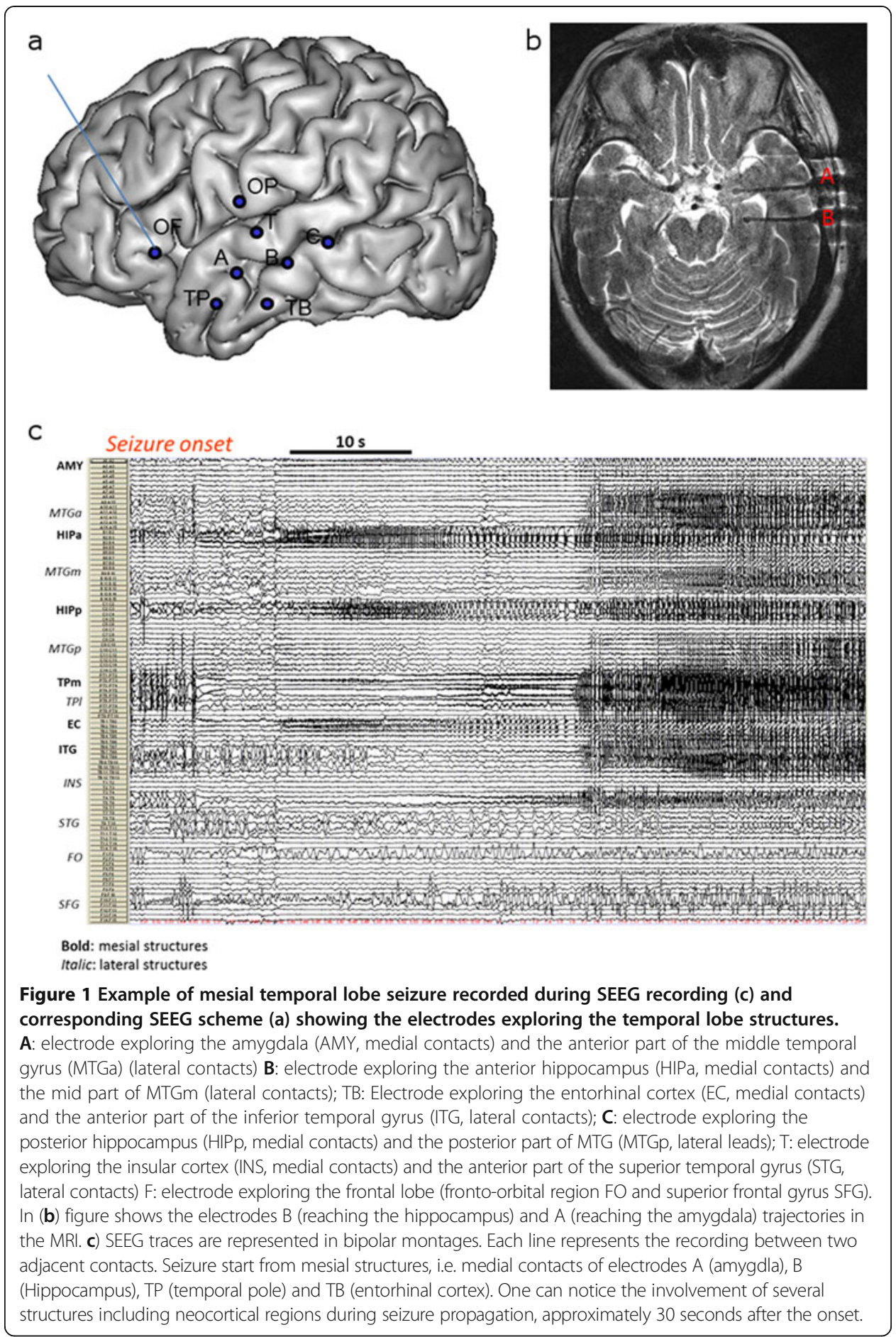

- the ability to synchronize their activity at seizure onset and during the course of the seizure.

LVRD constitute a characteristic electrophysiological pattern in focal seizures of human epilepsy characterized by a marked decrease of signal voltage (sometimes preceded by high-amplitude spikes) with a marked increase of signal frequency. They have 
long been observed in depth-EEG signals. Rapid discharges generally appear in brain regions in keeping with the epileptogenic zone [13] and are therefore considered as important marker providing substantial information about the EZ spatio-temporal organization [14,15]. The underlying neuronal mechanisms are not well known. Complex pattern of neuronal firing has been recently described during the initiation of seizures [12,16]. Computational modelisation [17] and in vivo studies [18] support the role of inhibitory neurons firing rather than an hyperactivity of principal cells in these phenomena. In temporal lobe seizures, the LVRD are generally less rapid disclosing a frequency peak in the beta or low gamma range, typically from 20 to $40 \mathrm{~Hz}$ [19-22].

\section{Synchronization and desynchronization in the epileptogenic zone network}

As indicated above, the seizure onset characterized by LVRD involves often distant and functionally distinct brain sites almost simultaneously. Thus it could be hypothesized that a "synchronizing phenomenon" gives rise to the simultaneous start of fast oscillations.

This hypothesis prompted us to study the spatio-temporal dynamics of these phenomena through measuring the interdependencies between generated signals.

It has been known for a long time that epileptic phenomena are associated with dramatic changes in brain synchrony mechanisms [23] and subsequent studies have shown that seizures in humans are associated with the abnormal synchronization of distant structures [24-26]. This synchronization can be quantified by measuring the interdependencies between signals recorded in different brain regions involved in the EZ. Numerous methods were proposed over the past decades, often categorized according to their ability to assess the linear (coherence, linear regression analysis) or nonlinear (mutual information, nonlinear regression analysis, similarity indexes based on statespace trajectories reconstructed from observed signals) properties of the relationship. Readers may refer to [27] for presentation of various kinds of methods and evaluation based on different models of relationship between signals (Figure 2). In brief, this study brought some insights about the behaviour of methods aimed at estimating functional brain connectivity from EEG signals. Both theoretical and technical features of aforementioned methods were comparatively addressed based on the use of three performance criteria. Interestingly, this study showed that none of the studied methods outperforms the other ones as the results provided by each method strongly depend on the underlying coupling model between analyzed time series. However, although they did not lead to the best results in all studied situations (coupled linear systems, phase/ amplitude couplings, coupled Rössler/Hénon nonlinear systems, coupled neural masses), regression analysis methods could always detect coupling changes. This was not the case for some other methods which were found to be blind in some studied situations. It is also worth mentioning that nonlinear regression analysis (see details below) was found to be particularly efficient to estimate functional connectivity from signals simulated from coupled neuronal populations (so called neural mass models). Such signals are known to well approximate actual local field potentials (LFPs) and SEEG signals, particularly regarding specific dynamics encountered in epileptogenic systems [17]. 




Using these methods, it is therefore possible to study functional couplings between several brain regions involved or not at seizure onset. The use of nonlinear approaches is probably well suited as it does not require assumptions on the nature or the relationship [28-30]. In this context, the so-called nonlinear regression analysis provides a parameter, referred to as the nonlinear correlation coefficient $h^{2}$, which takes values in $[0,1]$. Low values of $h^{2}$ denote that two signals $\mathrm{X}$ and $\mathrm{Y}$ under analysis are independent. On the other hand, high values of $\mathrm{h}^{2}$ mean that the second signal Y may be explained by a transformation (possibly nonlinear) of the first signal X (i.e. both signals are dependent).

In addition, this method offers the possibility to study the direction of the coupling between neuronal populations which is an important parameter to determine the "leader region" responsible for the "driving" input in the system. In addition to the estimation of $\mathrm{h}^{2}$, a second quantity has been proposed [29] that brings information on the causal property of the association. This quantity, referred to as the direction index D, takes into account both the estimated time delay $\tau$ between signals $\mathrm{X}$ and $\mathrm{Y}$ (latency) and the asymmetry property of the nonlinear correlation coefficient $h^{2}$ (values of the $h^{2}$ coefficient are different if the computation is performed from $\mathrm{X}$ to $\mathrm{Y}$ or from $\mathrm{Y}$ to $\mathrm{X}$ ). Values of parameter $\mathrm{D}$ range from -1.0 ( $\mathrm{X}$ is driven by $\mathrm{Y})$ to 1.0 ( $\mathrm{Y}$ is driven by $\mathrm{X}$ ). 
These methods in the last ten years have been mainly applied to the study of temporal lobe seizures (TLS) $[20,26,28,31,32]$. Finally, results of signal quantification demonstrate that the regions involved in the EZ establish preferential functional links during seizure and militate in favour of the existence of a network organization of the EZ.

In addition, the study of the statistical relations existing between SEEG signals at seizure onset, allowed us to identify 4 sub-types of TLS according to the interactions between mesial (amygdala-hippocampus-entorhinal cortex) and neocortical structures: mesial, mesial-lateral, and lateral-mesial and lateral [26,28]. Mesial TLS are the most frequent forms of TLS. In this group, functional coupling between several regions belonging to the mesial structures is observed. Absence of coupling between mesial and neocortical structures at seizure onset is also a characteristic feature of these seizures.

A more general phenomenon is that the coupling between brain structures is maximal before the emergence of the LVRD and tends to decrease after, to then increase once more later during the seizure course. This phenomenon is illustrated in Figure 3.

This "preictal" synchronization has been particularly quantified in a group of mesial temporal lobe epilepsy (MTLE), by studying the interactions between entorhinal cortex, hippocampus and amygdala [20]. Two main patterns of transition between interictal to ictal state may be observed in these seizures. Some seizures start with a fast discharge and seem to be particularly under the control of the entorhinal cortex while seizure starting by a "pre-ictal" periodic spiking are probably more likely triggered by the hippocampus. In vivo study of human epileptic tissue has shown that the preictal spiking is different from the interictal spiking. These events depend on glutamatergic mechanisms and are preceded by pyramidal cell firing, whereas interneuron firing precedes interictal events that depend on both glutamatergic and depolarizing GABAergic transmission. These pre-ictal discharges are involved in seizure initiation [16].

The desynchronization between signals observed after a first phase of synchrony, was found to be particularly important when we studied the signal from neocortical sites in neocortical seizures [14]. This desynchronization has been also observed in both neural activity and local field potentials in vitro and in vivo [33,34]. Finally, the pattern of synchronization/desynchronization between regions forming the EZ appears to be a characteristic property of the EZ and may be observed in many different forms of partial seizures.

\section{The role of synchronization/desynchronization in clinical manifestations}

Video-SEEG recordings are classically used to make anatomo-electro-clinical correlations and to determine which regions of the brain are responsible for clinical symptoms. One of the most striking phenomena is that semiology generally appears when a large set of different structures are involved in the ictal discharge. In addition, the first clinical signs are observed after the seizure onset (typically several seconds) and are largely related to the propagation of the discharge.

Coordinated interactions between neurons and neuronal populations are an essential feature of brain functioning [35]. In the healthy brain, cognitive and emotional processes are dependent on precise integration of neural activity at specific spatiotemporal scales [36-38].

We made the hypothesis that signs and symptoms during the seizure course could be related to changes and new interactions taking place between brain regions during the 

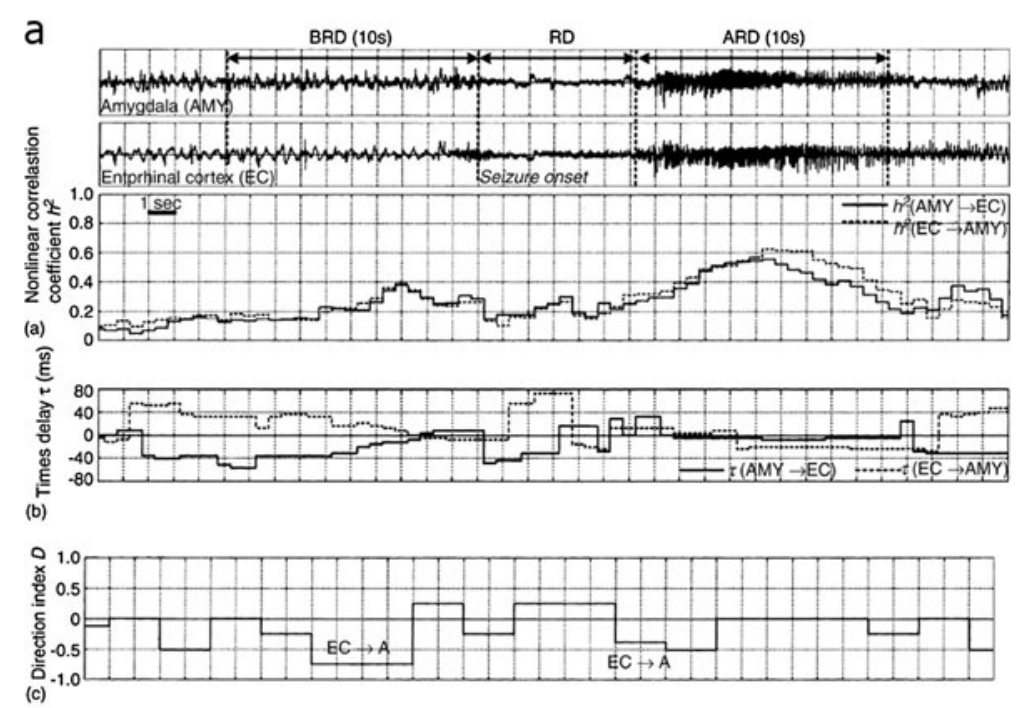

b

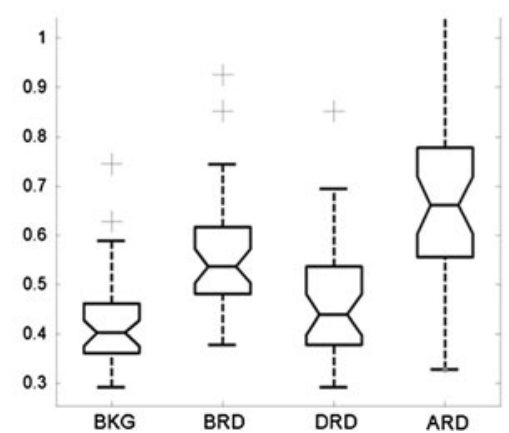

Figure 3 SEEG functional connectivity in TLS. a. A study of functional coupling between entorhinal cortex and amygdala in a patient with mesial temporal lobe seizures. An increase of synchrony (1) during the phase of preictal spiking is observed as measured by the coefficient $h^{2}$ between the two structures. The direction index $\mathrm{D}$ indicates that the activity in the entorhinal cortex is leading (negative values) that recorded in the amygdala. Note that the rapid discharge is associated with a decrease of correlation (reproduced from [20]). b. Boxplot performed on normalized values of correlations $\left(h^{2}\right)$ between signals recorded from amygdala, entorhinal cortex and hippocampus during SEEG recorded mesial temporal lobe seizures. The lower and upper lines of each "box" (25th and 75th percentiles of each sample) and the line in the middle of the box (sample median) respectively indicate that the distributions of $h^{2}$ values over the $B K G, B R D$ and DRD periods are narrower than that computed over the ARD period. $h^{2}$ values measured before the rapid discharge (BRD) are significantly higher than those measured during background activity (BKG) and during the rapid discharge itself (DRD). For the period that follows the rapid discharge (ARD) strong re-coupling is indicated by significantly high $h^{2}$ values (adapted from [20]).

development of ictal rhythms. Unfortunately, so far very few studies have attempted to correlate symptoms to changes in brain synchronization. In recent years, we have suggested that some symptoms could be related to the uncontrolled activation of functionally-normal neural networks by epileptic rhythms or on the contrary be related to the disruption of mechanisms underlying normal brain function.

The former situation can be seen in the building of some "elaborated" symptoms mimicking normal behaviours. One of the most remarkable examples is the "dreamy state" that encompasses two kinds of phenomenona: an illusion of familiarity (déjà vu-déjà vécu) or a reminiscence of visual memories. For a long time, the anatomical localization and the mechanisms of these phenomena have been the object of impassioned debates [39]. At the time being, it is admitted that these phenomena are related to epileptic discharges 
involving the memory systems of the mesial temporal lobe. We recently showed that these "dysmnesic" phenomena were more frequent after stimulation of the rhinal cortices than after stimulation of the hippocampus or the amygdala [40]. We particularly studied one of the patients from this series, in whom the stimulation of perirhinal cortex provoked the recollection of vivid visual scenes. The interdependencies existing between signals generated at the time of the phenomenon of memory recollection were quantified by linear correlation between intracerebral EEG signals filtered into classical frequency sub-bands. We demonstrated that a transient theta range synchronization (studied using linear correlation measure) between the mesial temporal lobe structures as well as a primary visual area was observed at the time of the reminiscence [41]. These results are thus suggestive of the transient activation of a neural network normally involved in the recall of memories.

More recently, we analyzed synchronization of intracerebral EEG signals recorded from mesial temporal lobe structures in epileptic patients in which "déjà vu" was induced by electrical stimulation. In comparison with stimulations that did not lead to "déjà vu" stimulations triggering "déjà vu" were associated with increased EEG correlation that was particularly significant in the theta band (Figure 4a). Results are in favour of a mechanism involving transient cooperation between various MTL structures including hippocampus and entorhinal cortex [42].

Another example is the humming/singing automatism. Some patients during temporal seizures exhibit a humming/singing behaviour, highly reproducible from one seizure to

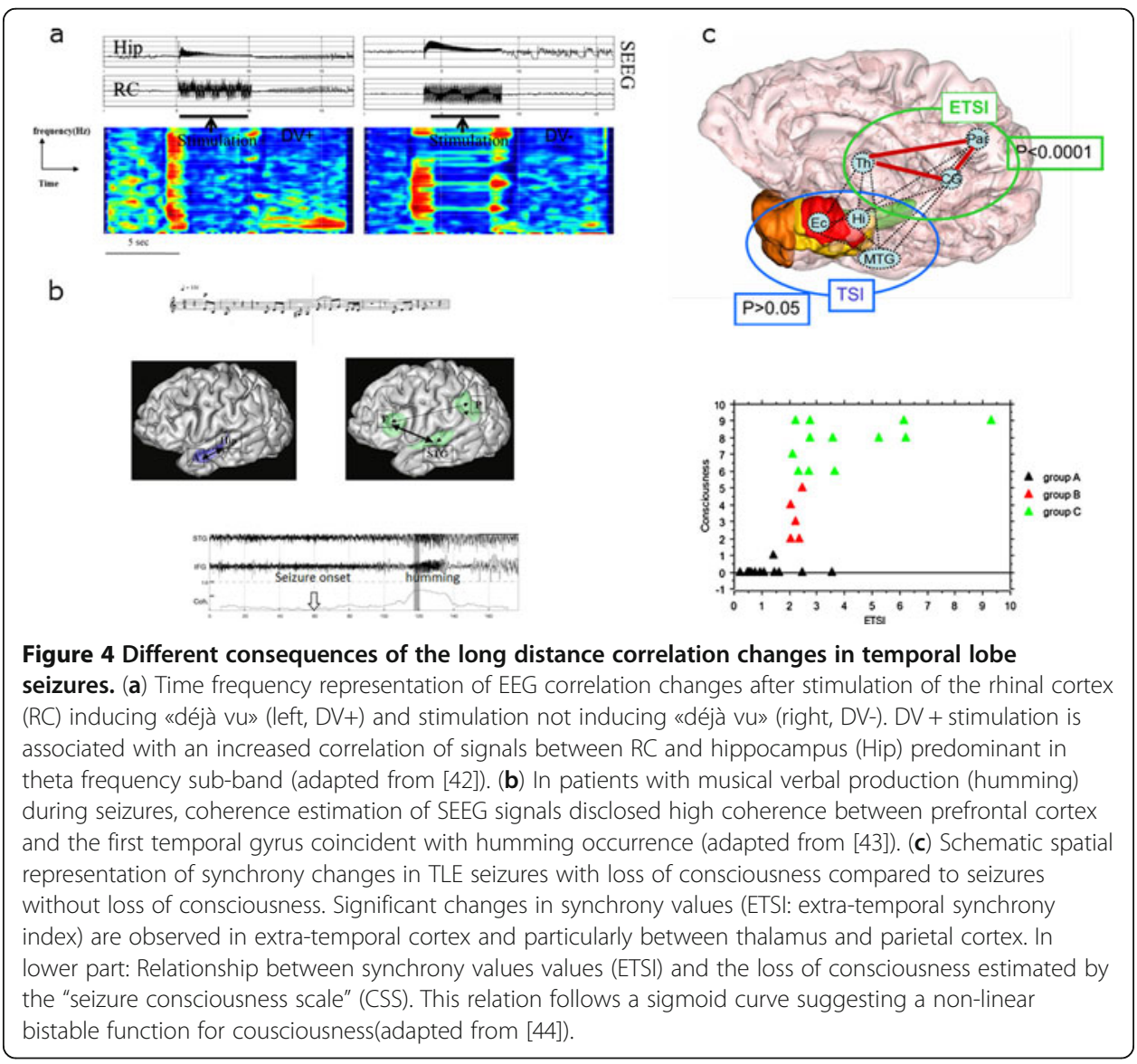


another. We studied three patients explored by SEEG and presenting with such an automatism [43]. In comparison with the seizure onset period, we showed that a functional coupling (characterized by the coherence averaged over the frequency band) appears between the temporal superior gyrus and the prefrontal cortex during the humming phenomenon (Figure $4 \mathrm{~b}$ ).

These examples are remarkable in that they suggest that ictal activity, observable in areas remote from the EZ, is able to "reactivate" neural networks involved in some specific (memory for dreamy state, musical function) brain functions.

Excess of synchronization may be in contrast deleterious for brain functioning, particularly for conscious representations. In a recent study, we studied the relationship between neural synchronization and loss of consciousness in MTLE seizures [6]. Loss of consciousness (LOC) is a dramatic clinical manifestation of temporal lobe seizures. Its underlying mechanism could involve altered coordinated neuronal activity between the brain regions that support conscious information processing [45]. The consciousness access hypothesis assumes the existence of a global workspace in which information becomes available via synchronized activity within neuronal modules, often widely distributed throughout the brain [46,47]. Re-entry loops and, in particular, thalamocortical communication would be crucial to functionally bind together different modules. We used intracranial recordings of cortical and subcortical structures in 12 patients with intractable temporal lobe epilepsy as part of their presurgical evaluation to investigate the relationship between states of consciousness and neuronal activity within the brain $[6,48]$. The synchronization of EEG signals between distant regions was estimated as a function of time by using non-linear regression analysis. We found that LOC occurring during TLS is characterized by increased long-distance synchronization between structures that are critical in processing awareness, including thalamus and parietal cortices (Figure 4c). The degree of LOC was found to correlate with the amount of synchronization in thalamo-cortical systems. This result suggested that excessive synchronization may overload the structures involved in consciousness processing, preventing them from treating incoming information and thus resulting in LOC. The transition between consciousness and loss of consciousness as a function of synchronization followed a non linear curve, suggesting a bi-stable system: above a certain threshold of synchronization, the involved brain networks are unable to process consciousness representation. This result has been recently extended to extratemporal seizures [49].

Desynchronization could also be important for determining ictal symptoms, particularly during complex emotional behaviours (seizures are marked by screaming, agitation, terror expression) [50]. In these cases, the symptoms are correlated with the rapid ictal discharge affecting different "para-limbic" regions of both frontal and temporal lobes [51]. Study of correlations between signals have shown that these intense emotional alterations were associated with a decrease of synchrony [50] between signals recorded from the neural networks known to be involved in emotional processing, and in particular a loss of correlations between the orbito-frontal cortex and the amygdala. Since orbitofrontal cortex is an important regions regulating the amygdala and related subcortical regions, this disruption of functional connections could then result in the disruption of emotional regulation leading to the release of altered behaviour (Figure 5). 




\section{Involvement of thalamo-cortical synchrony in temporal lobe epilepsy (TLE) seizures}

The anatomy of TLE seizures has been particularly studied from human electroencephalographic (EEG) recordings as well as from animal studies using induced temporal lobe seizures. However, structures outside the temporal lobe might also play a role in temporal lobe seizures. A potentially important extra-temporal structure that could be involved early in the temporal organization of MTLE seizures is the midline thalamus (TH). The role of synchronized thalamo-cortical oscillations has been well demonstrated in animal models of absence seizures [30]. During partial seizures, the role of such interactions has been less studied. Electrophysiological recordings have shown that midline TH may be early involved in spontaneous seizures of chronic epileptic rats [52]. It has thus been suggested that the $\mathrm{TH}$ could act as a seizure amplifier and synchronizer of the ictal activity. Thalamic activity during TLE seizures (using intracerebral recordings, SEEG technique) has been reported [5,53]. The synchronization between signals from temporal lobe structures and the $\mathrm{TH}$ has been investigated in human TLE [5]. The results demonstrated an overall increase of correlation between $\mathrm{TH}$ and temporal lobe structures during seizures (Figure 6). Correlation values from seizure onset period were significantly higher than values from the background period $(p=0.001)$. Values at the end of seizure were significantly higher than values from the 


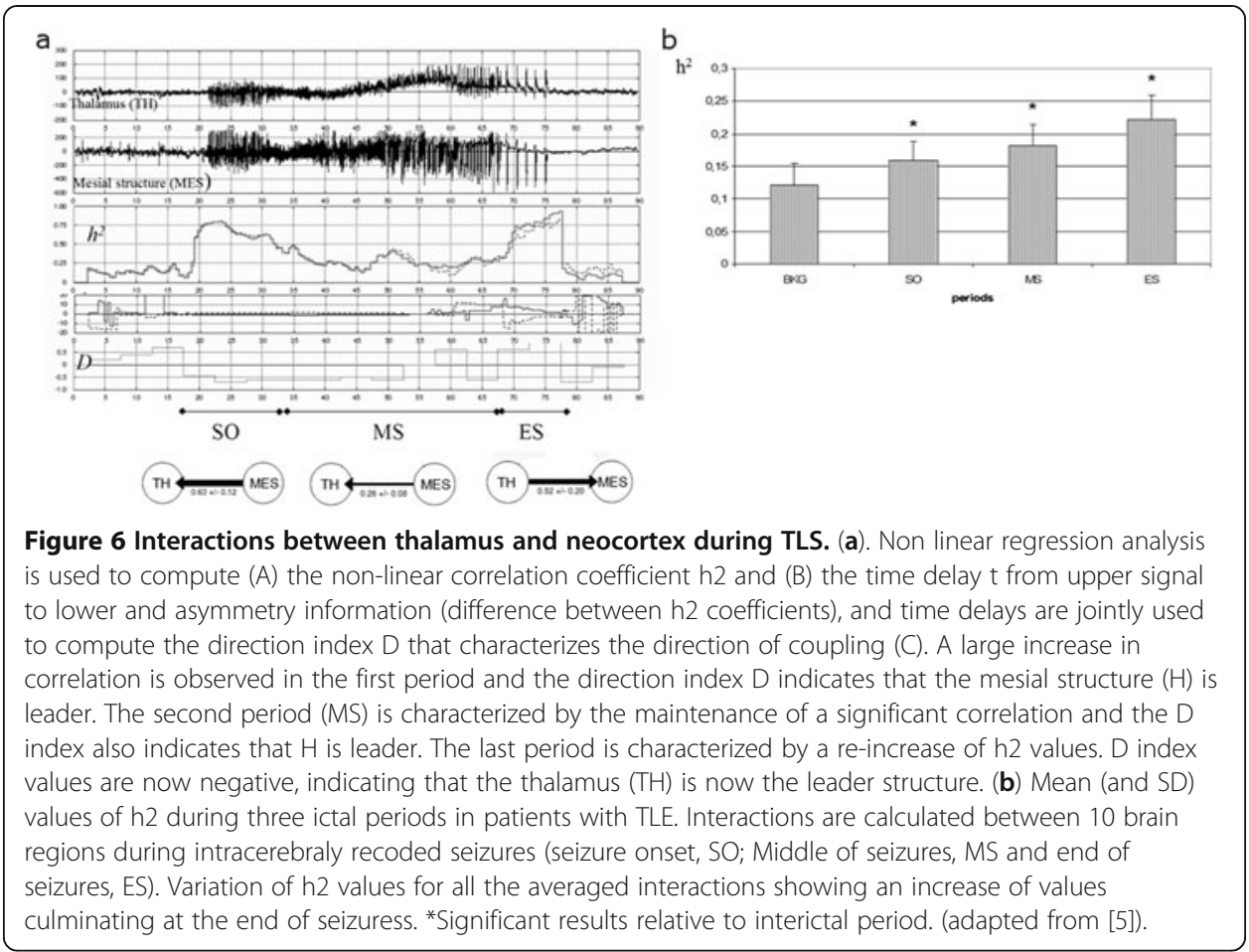

seizure onset $(\mathrm{p}<0.0001)$. Thus, thalamo-cortical synchronization was found to predominate at the end of the seizures, a period of maximal values of correlations.

Interestingly the termination of seizures has been proposed to be caused by the large increase in signal synchrony observed at the end of seizures [54,55]. This report demonstrated that the $\mathrm{TH}$ and remote cortical structures synchronize their activity during TLE seizures and suggested that the extension of the epileptogenic network to the TH is a potential important factor determining surgical prognosis.

\section{The epileptogenic zone discloses altered synchrony during the interictal state}

Despite the classical notion stipulating that epilepsy is associated with abnormally-high synchrony within and between neuronal populations, there are in fact very few works that have proved that during the interictal state, the epileptic brain is the seat of altered synchronization between distant neuronal assemblies. The study reported in [56] showed a local increase in interictal synchrony using the mean phase coherence in a group of 17 MTLE patients. A recent study estimated the interdependencies between signals recorded by subdural grids in nine patients during presurgical evaluation with neocortical epilepsy [57]. Inter-electrode synchrony was quantified using the mean phase coherence algorithm and revealed areas of elevated local synchrony that may be a marker of epileptogenic cortex.

In a recent study [58] we characterized the functional coupling between mesial structures during the interictal state by comparing the MTLE group to a "control" group including patients with extra-temporal seizures. Results showed that functional coupling was enhanced in the epileptogenic zone in this group of patients (MTLE). This effect 
was significant for the theta $(4-8 \mathrm{~Hz})$, alpha $(8-12 \mathrm{~Hz})$, beta $(12-25 \mathrm{~Hz})$ and gamma bands $(25-90 \mathrm{~Hz})$ and to a large part independent of the occurrence of interictal spiking. The functional significance of an increase of EEG synchrony between structures forming the EZ is unclear. We can assume that even during the interictal state, abnormal longrange reinforced EEG connectivity between structures forming the EZ network is a fundamental characteristic of this region. Of particular interest, in a recent study using the amygdala kindling model in the rat (an animal model of secondary epileptogenesis), an increase of coherence values between amygdala and frontal cortex was observed. Therefore, enhanced connectivity appears to be a marker of the kindling phenomenon at the EEG level [59]. This result suggests that this particular state can "facilitate" seizure occurrence by "priming" the system to synchronize during seizures.

During the interictal period, we have also investigated the functional connectivity (FC) within and outside the EZ by using resting-state functional MRI (rsfMRI). Interestingly, in a first study we found a decreased FC within the epileptogenic networks in comparison to a control group contrasting with our results by using SEEG [60]. Conversely we found increased FC in the contralateral MTL structures that correlated to cognitive performances. These results were confirmed in a further study conducted in a larger cohort in which we also demonstrated the potential usefulness of rsfMRI in the lateralization of the EZ at an individual level [61]. Finally, in a last study, we confirmed the apparent paradoxical pattern of FC as measured by SEEG and rsfMRI by co-registering the site of recording and the region of interest chosen for computing FC on rsfMRI data in the same cohort of patients [62]. We proposed different non contradictory hypothesis to interpret this apparent discrepancy between increased FC as measured by SEEG and decreased FC as measured by rsfMRI within the epileptogenic networks. The first is a potential neurovascular decoupling in a pathological network affected by metabolic, perfusion and blood-

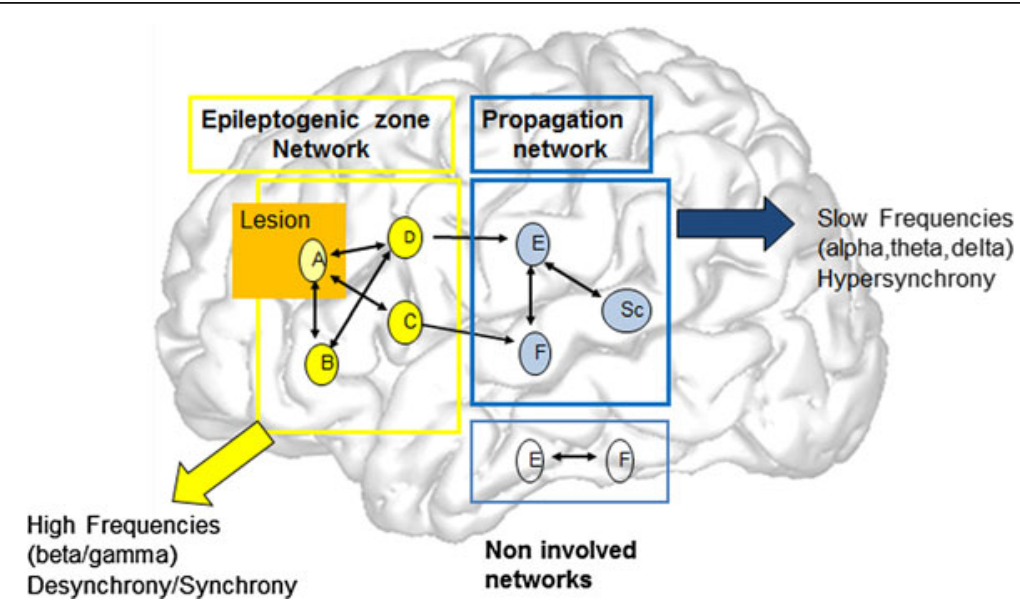

Figure $7 \mathrm{~A}$ model of the network organization of partial seizures. The brain structures are represented by letters (A,B, etc.). The scheme proposes the existence of two groups of structures in the epileptic brain. Some of them are able to generate seizures, and particularly fast discharges at seizure onset. They constitue the EZ network (labelled $A, B, C, D$ ). A represents a region with putative (visible) lesion. The EZ network produces rapid discharge and is characterized by a pattern of Synchrony-Desynchrony. A second set of structures are less epileptogenic and are triggered in seizures by the EZ ("propagation zone network", structures (E,F,SC, H). SC correspond to subcortical (thalamus for instance) regions. Activity recorded in these regions are of lower frequency and generally more synchronized than in the EZ. The Propagation zone network can in turn synchronize with the EZ network during the course of the seizure. 
brain-barrier alterations, especially in the type of partial epilepsies we explored (i.e. temporal lobe epilepsy). The second is that each technique actually quantifies different epilepsy-related phenomena potentially occurring at different time scales and dynamic, affecting correlations between signals in different ways. These results demonstrate the complexity of signal coupling even during the interictal state. Functional connectivity measured between seizures by EEG signal is therefore representative of pathological electrical signal interaction [57] whereas disrupted functional connectivity measured in the same period by BOLD is rather representative of dysfunction [63-66].

Interestingly, studies dedicated to structural connectivity in epilepsy using either morphometric correlation or diffusion imaging - tractography have also demonstrated a decreased structural connectivity affecting major bundles connected to the epileptogenic regions and even beyond [67-71]. We hypothesize that this anatomical disconnection can also play a role in the decrease of FC as measured by fMRI. We also think that this structural disconnection may have a greater impact on BOLD signal correlation than on SEEG signal interdependencies in pathological regions.

Interestingly, we also quantified the causal relationships (effective connectivity) between three kinds of networks labeled according to SEEG results (i.e. the epileptogenic zone network, the propagation network and network non-involved by electrical abnormalities) [62]. Surprisingly, we found an influence of the epileptogenic network on the "non-involved" network spared by electrical abnormalities but potentially driven by epileptic regions.

\section{Conclusions}

A general scheme of the organization of human partial seizures can be outlined from the aforementioned studies and is illustrated in Figure 7 [4,10,72]. Seizures are generated in an initial network of epileptogenic brain structures (A,B,C,D) (epileptogenic zone network, $E Z$ network) whose activity is transiently synchronized and after desynchronized with the appearance of fast oscillations. This network may include a lesion (A, yellow circle). During a second ictal phase, other cortical (E, F) and subcortical structures (SC) are the seat of slower rhythmic modifications that are synchronized (propagation network). The emergence of a particular clinical semiology in the course of the seizure depends on these phenomena which can in certain cases "mimick" a normal cerebral process or on the contrary provoke a major rupture in normal cerebral functioning.

Competing interests

The authors declare that they have no competing interests.

Authors' contribution

FB,FW and MG have written the paper and all authors have given final approval for its publication. All authors read and approved the final manuscript.

\section{Author details}

${ }^{1}$ INSERM, U1106, Marseille F-13000, France. ²Faculté de Médecine, Aix Marseille Université, Marseille F-13000, France.

${ }^{3}$ Assistance Publique - Hôpitaux de Marseille, Hôpital de la Timone, Service de Neurophysiologie Clinique, CHU

Timone-264 Rue st Pierre, Marseille F-13000, France. ${ }^{4}$ INSERM, U1099, Rennes F-35000, France. 5 LTSI, Université de Rennes 1, Rennes F-35000, France. ${ }^{6}$ Centre de Resonance Magnetique Biologique et Medicale UMR CNRS 7339,

Marseille F-13000, France. ${ }^{7}$ Assistance Publique - Hôpitaux de Marseille, Hôpital de la Timone, CEMEREM, Marseille F-13000, France. 
References

1. Jiruska P, de Curtis M, Jefferys JG, Schevon CA, Schiff SJ, Schindler K: Synchronization and desynchronization in epilepsy: controversies and hypotheses. J Physio/ 2013, 591:787-797.

2. Uhlhaas PJ, Singer W: Neural synchrony in brain disorders: relevance for cognitive dysfunctions and pathophysiology. Neuron 2006, 52:155-168.

3. Wendling F, Chauvel P, Biraben A, Bartolomei F: From intracerebral EEG signals to brain connectivity: identification of epileptogenic networks in partial epilepsy. Frontiers Syst Neurosci 2010, 4:154.

4. Bartolomei F, Wendling F: Synchrony in neural networks underlying seizure generation in human partial epilepsies. In Coordinated activity in the brain: measurements and relevance to brain function and behavior. Edited by Velazquez J, Wennberg R. New York: Springer; 2009:137-147.

5. Guye M, Regis J, Tamura M, Wendling F, McGonigal A, Chauvel P, Bartolomei F: The role of corticothalamic coupling in human temporal lobe epilepsy. Brain 2006, 129:1917-1928.

6. Arthuis M, Valton L, Regis J, Chauvel P, Wendling F, Naccache L, Bernard C, Bartolomei F: Impaired consciousness during temporal lobe seizures is related to increased long-distance cortical-subcortical synchronization. Brain 2009, 132:2091-2101.

7. Ponten S, Bartolomei F, Stam C: Small-world networks and epilepsy: graph theoretical analysis of intracerebrally recorded mesial temporal lobe seizures. Clin Neurophysio/ 2007. doi:10.1016/j. clinph.2006.12.002.

8. Kramer MA, Kolaczyk ED, Kirsch HE: Emergent network topology at seizure onset in humans. Epilepsy Res 2008, 2-3:173-186.

9. Rosenow F, Luders H: Presurgical evaluation of epilepsy. Brain 2001, 124:1683-1700.

10. Bartolomei F, Chauvel P, Wendling F: Spatio-temporal dynamics of neuronal networks in partial epilepsy. Rev Neurol (Paris) 2005, 161:767-780

11. Wendling F, Badier J, Chauvel P, Coatrieux J: A method to quantify invariant information in depth-recorded epileptic seizures. Electroenceph Clin Neurophysiol 1997, 102:472-485.

12. Truccolo W, Donoghue JA, Hochberg LR, Eskandar EN, Madsen JR, Anderson WS, Brown EN, Halgren E, Cash SS: Single-neuron dynamics in human focal epilepsy. Nat Neurosci 2011, 14:635-641.

13. Bancaud J, Angelergues R, Bernouilli C, Bonis A, Bordas-Ferrer M, Bresson M, Buser P, Covello L, Morel P, Szikla G, et al: Functional stereotaxic exploration (SEEG) of epilepsy. Electroencephalogr Clin Neurophysiol 1970, 28:85-86.

14. Wendling F, Bartolomei F, Bellanger JJ, Bourien J, Chauvel P: Epileptic fast intracerebral EEG activity: evidence for spatial decorrelation at seizure onset. Brain 2003, 126:1449-1459.

15. Alarcon G, Binnie CD, Elwes RD, Polkey CE: Power spectrum and intracranial EEG patterns at seizure onset in partial epilepsy. Electroencephalogr Clin Neurophysiol 1995, 94:326-337.

16. Huberfeld G, Menendez de la Prida L, Pallud J, Cohen I, Le Van Quyen M, Adam C, Clemenceau S, Baulac M, Miles R: Glutamatergic pre-ictal discharges emerge at the transition to seizure in human epilepsy. Nat Neurosci 2011, 14:627-634.

17. Wendling F, Hernandez A, Bellanger JJ, Chauvel P, Bartolomei F: Interictal to ictal transition in human temporal lobe epilepsy: insights from a computational model of intracerebral EEG. J Clin Neurophysiol 2005, 22:343-356.

18. Gnatkovsky V, Librizzi L, Trombin F, de Curtis M: Fast activity at seizure onset is mediated by inhibitory circuits in the entorhinal cortex in vitro. Ann Neurol 2008, 64:674-686.

19. Javidan M, Katz A, Tran T, Pacia S, Spencer D, Spencer S: Frequency characteristics of neocortical and hippocampal onset seizures. Epilepsia 1992, 33(Suppl 3):58.

20. Bartolomei F, Wendling F, Regis J, Gavaret M, Guye M, Chauvel P: Pre-ictal synchronicity in limbic networks of mesial temporal lobe epilepsy. Epilepsy Res 2004, 61:89-104.

21. Bartolomei F, Chauvel P, Wendling F: Epileptogenicity of brain structures in human temporal lobe epilepsy: a quantified study from intracerebral EEG. Brain 2008, 131:1818-1830.

22. Aubert S, Wendling F, Regis J, McGonigal A, Figarella-Branger D, Peragut JC, Girard N, Chauvel P, Bartolomei F: Local and remote epileptogenicity in focal cortical dysplasias and neurodevelopmental tumours. Brain 2009, 132:3072-3086.

23. Brazier MA: Spread of seizure discharges in epilepsy: anatomical and electrophysiological considerations. Exp Neurol 1972, 36:263-272.

24. Gotman J, Levtova V: Amygdala-hippocampus relationships in temporal lobe seizures: a phase coherence study. Epilepsy Res 1996, 25:51-57.

25. Le Van Quyen M, Adam C, Baulac M, Martinerie J, Varela F: Nonlinear interdependencies of EEG signals in human intracranially recorded temporal lobe seizures. Brain Res 1998, 792:24-40.

26. Bartolomei F, Wendling F, Vignal J, Kochen S, Bellanger J, Badier J, Le Bouquin-Jeannes R, Chauvel P: Seizures of temporal lobe epilepsy: identification of subtypes by coherence analysis using stereo-electro-encephalography. Clin Neurophysiol 1999, 110:1741-1754.

27. Wendling F, Ansari-As| K, Bartolomei F, Senhadji L: From EEG signals to brain connectivity: a model-based evaluation of interdependence measures. J Neurosci Methods 2009, 30(183):9-18.

28. Bartolomei F, Wendling F, Bellanger J, Regis J, Chauvel P: Neural networks involved in temporal lobe seizures: a nonlinear regression analysis of SEEG signals interdependencies. Clin Neurophysiol 2001, 112:1746-1760.

29. Wendling F, Bartolomei F: Modeling EEG signals and interpreting measures of relationship during temporallobe seizures: an approach to the study of epileptogenic networks. Epileptic Disord 2001, Special Issue:67-78

30. Meeren HK, Pijn JP, Van Luijtelaar EL, Coenen AM, Lopes da Silva FH: Cortical focus drives widespread corticothalamic networks during spontaneous absence seizures in rats. J Neurosci 2002, 22:1480-1495.

31. Bartolomei F, Khalil M, Wendling F, Sontheimer A, Regis J, Ranjeva JP, Guye M, Chauvel P: Entorhinal cortex involvement in human mesial temporal lobe epilepsy: an electrophysiologic and volumetric study. Epilepsia 2005, 46:677-687.

32. Wendling F, Bartolomei F, Bellanger JJ, Chauvel P: Interpretation of interdependencies in epileptic signals using a macroscopic physiological model of the EEG. Clin Neurophysiol 2001, 112:1201-1218. 
33. Netoff TI, Schiff SJ: Decreased neuronal synchronization during experimental seizures. J Neurosci 2002, 22:7297-7307.

34. Cymerblit-Sabba A, Schiller Y: Development of hypersynchrony in the cortical network during chemoconvulsant-induced epileptic seizures in vivo. J Neurophysiol 2012, 107:1718-1730.

35. Velazquez J, Guevara Erra R, Wennberg R, Dominguez L: Correlations of cellular activities in the nervous system: physiological and methodological considerations. In Coordinated activity in the brain: measurements and relevance to brain function and behavior. Edited by Velazquez J, Wennberg R. New York: Springer; 2009:1-24.

36. Varela F, Lachaux JP, Rodriguez E, Martinerie J: The brainweb: phase synchronization and large-scale integration. Nat Rev Neurosci 2001, 2:229-239.

37. Stam CJ, van Straaten EC: The organization of physiological brain networks. Clin Neurophysiol 2012, 123:1067-1087.

38. Bassett DS, Bullmore ET, Meyer-Lindenberg A, Apud JA, Weinberger DR, Coppola R: Cognitive fitness of cost-efficient brain functional networks. Proc Natl Acad Sci U S A 2009, 106:11747-11752.

39. Bancaud J, Brunet-Bourgin F, Chauvel P, Halgren E: Anatomical origin of déjà vu and vivid 'memories' in human temporal lobe epilepsy. Brain 1994, 117:71-90.

40. Bartolomei F, Barbeau E, Gavaret M, Guye M, McGonigal A, Regis J, Chauvel P: Cortical stimulation study of the role of rhinal cortex in deja vu and reminiscence of memories. Neurology 2004, 63:858-864

41. Barbeau E, Wendling F, Regis J, Duncan R, Poncet M, Chauvel P, Bartolomei F: Recollection of vivid memories after perirhinal region stimulations: synchronization in the theta range of spatially distributed brain areas. Neuropsychologia 2005, 43:1329-1337.

42. Bartolomei F, Barbeau EJ, Nguyen T, McGonigal A, Regis J, Chauvel P, Wendling F: Rhinal-hippocampal interactions during deja vu. Clin Neurophysiol 2012, 123:489-495.

43. Bartolomei F, Wendling F, Vignal JP, Chauvel P, Liegeois-Chauvel C: Neural networks underlying epileptic humming. Epilepsia 2002, 43:1001-1012.

44. Arthuis M, Valton L, Regis J, Chauvel P, Wendling F, Naccache L, Bernard C, Bartolomei F: Impaired consciousness during temporal lobe seizures is related to increased long-distance cortical-subcortical synchronization. Brain 2009, 132:2091-2101.

45. Dehaene S, Naccache L: Towards a cognitive neuroscience of consciousness: basic evidence and a workspace framework. Cognition 2001, 79:1-37.

46. Bartolomei F, Naccache L: The global workspace (GW) theory of consciousness and epilepsy. Behav Neurol 2011, 24:67-74.

47. Sergent C, Naccache L: Imaging neural signatures of consciousness: 'What', 'When', 'Where' and 'How' does it work? Archives italiennes de biologie 2012, 150:91-106.

48. Bartolomei F: Coherent neural activity and brain synchronization during. Archives italiennes de biologie 2012 150:164-171.

49. Lambert I, Arthuis M, McGonigal A, Wendling F, Bartolomei F: Alteration of global workspace during loss of consciousness: a study of parietal seizures. Epilepsia 2012, 53:2104-2110.

50. Bartolomei F, Trebuchon A, Gavaret M, Regis J, Wendling F, Chauvel P: Acute alteration of emotional behaviour in epileptic seizures is related to transient desynchrony in emotion-regulation networks. Clin Neurophysiol 2005, 116:2473-2479.

51. Vaugier L, Aubert S, McGonigal A, Trebuchon A, Guye M, Gavaret M, Regis J, Chauvel P, Wendling F, Bartolomei F: Neural networks underlying hyperkinetic seizures of "temporal lobe" origin. Epilepsy Res 2009, 86:200-208.

52. Bertram EH, Mangan PS, Zhang D, Scott CA, Williamson JM: The midline thalamus: alterations and a potential role in limbic epilepsy. Epilepsia 2001, 42:967-978.

53. Rosenberg DS, Mauguiere F, Demarquay G, Ryvlin P, Isnard J, Fischer C, Guenot M, Magnin M: Involvement of medial pulvinar thalamic nucleus in human temporal lobe seizures. Epilepsia 2006, 47:98-107.

54. Topolnik L, Steriade M, Timofeev I: Partial cortical deafferentation promotes development of paroxysmal activity. Cereb Cortex 2003, 13:883-893.

55. Schindler $\mathrm{K}$, Leung $\mathrm{H}$, Elger $C E$, Lehnertz $\mathrm{K}$ : Assessing seizure dynamics by analysing the correlation structure of multichannel intracranial EEG. Brain 2007, 130:65-77.

56. Mormann F, Lehnertz K, David P, Elger CE: Mean phase coherence as a measure for phase synchronization and its application to the EEG of epilepsy patients. Physica D 2000, 144:358-369.

57. Schevon CA, Cappell J, Emerson R, Isler J, Grieve P, Goodman R, McKhann G Jr, Weiner H, Doyle W, Kuzniecky R, et al: Cortical abnormalities in epilepsy revealed by local EEG synchrony. Neuroimage 2007, 35:140-148.

58. Bettus G, Wendling F, Guye M, Valton L, Regis J, Chauvel P, Bartolomei F: Enhanced EEG functional connectivity in mesial temporal lobe epilepsy. Epilepsy Res 2008, 81:58-68.

59. Blumenfeld H, Rivera M, Vasquez JG, Shah A, Ismail D, Enev M, Zaveri HP: Neocortical and thalamic spread of amygdala kindled seizures. Epilepsia 2007, 48:254-262.

60. Bettus G, Guedj E, Joyeux F, Confort-Gouny S, Soulier E, Laguitton V, Cozzone PJ, Chauvel P, Ranjeva JP, Bartolomei F, Guye M: Decreased basal fMRI functional connectivity in epileptogenic networks and contralateral compensatory mechanisms. Hum Brain Mapp 2009, 30:1580-1591.

61. Bettus G, Bartolomei F, Confort-Gouny S, Guedj E, Chauvel P, Cozzone PJ, Ranjeva JP, Guye M: Role of resting state functional connectivity MRI in presurgical investigation of mesial temporal lobe epilepsy. J Neurol Neurosurg Psychiatry 2010, 81:1147-1154.

62. Bettus G, Ranjeva JP, Wendling F, Benar CG, Confort-Gouny S, Regis J, Chauvel P, Cozzone PJ, Lemieux L, Bartolomei F, Guye M: Interictal functional connectivity of human epileptic networks assessed by intracerebral EEG and BOLD signal fluctuations. PLOS One 2011, 6:e20071.

63. Waites AB, Briellmann RS, Saling MM, Abbott DF, Jackson GD: Functional connectivity networks are disrupted in left temporal lobe epilepsy. Ann Neurol 2006, 59:335-343.

64. Liao W, Zhang Z, Pan Z, Mantini D, Ding J, Duan X, Luo C, Lu G, Chen H: Altered functional connectivity and small-world in mesial temporal lobe epilepsy. PLoS One 2010, 5:e8525. 
65. Zhang Z, Lu G, Zhong Y, Tan Q, Liao W, Wang Z, Wang Z, Li K, Chen H, Liu Y: Altered spontaneous neuronal activity of the default-mode network in mesial temporal lobe epilepsy. Brain Res 2010, 1323:152-160.

66. Vlooswijk MC, Jansen JF, Majoie HJ, Hofman PA, de Krom MC, Aldenkamp AP, Backes WH: Functional connectivity and language impairment in cryptogenic localization-related epilepsy. Neurology 2010, 75:395-402.

67. Bonilha L, Edwards JC, Kinsman SL, Morgan PS, Fridriksson J, Rorden C, Rumboldt Z, Roberts DR, Eckert MA, Halford JJ: Extrahippocampal gray matter loss and hippocampal deafferentation in patients with temporal lobe epilepsy. Epilepsia 2010, 51:519-528.

68. Powell HW, Parker GJ, Alexander DC, Symms MR, Boulby PA, Wheeler-Kingshott CA, Barker GJ, Koepp MJ, Duncan JS: Abnormalities of language networks in temporal lobe epilepsy. Neuroimage 2007, 36:209-221.

69. Yogarajah M, Powell HW, Parker GJ, Alexander DC, Thompson PJ, Symms MR, Boulby P, Wheeler-Kingshott CA, Barker GJ, Koepp MJ, Duncan JS: Tractography of the parahippocampal gyrus and material specific memory impairment in unilateral temporal lobe epilepsy. Neuroimage 2008, 40:1755-1764.

70. McDonald CR, Ahmadi ME, Hagler DJ, Tecoma ES, Iragui VJ, Gharapetian L, Dale AM, Halgren E: Diffusion tensor imaging correlates of memory and language impairments in temporal lobe epilepsy. Neurology 2008, 71:1869-1876.

71. Bernhardt BC, Worsley K, Besson P, Concha L, Lerch JP, Evans AC, Bernasconi N: Mapping limbic network organization in temporal lobe epilepsy using morphometric correlations: insights on the relation between mesiotemporal connectivity and cortical atrophy. Neuroimage 2008, 42:515-524.

72. Bartolomei F, Wendling F, Chauvel P: The concept of an epileptogenic network in human partial epilepsies. Neurochirurgie 2008, 54:174-184.

doi:10.1140/epjnbp11

Cite this article as: Bartolomei et al:: Abnormal binding and disruption in large scale networks involved in human partial seizures. EPJ Nonlinear Biomedical Physics 2013 1:4

\section{Submit your manuscript to a SpringerOpen ${ }^{\circ}$ journal and benefit from:}

- Convenient online submission

- Rigorous peer review

- Immediate publication on acceptance

- Open access: articles freely available online

- High visibility within the field

- Retaining the copyright to your article 